\title{
The therapeutic potential of immune cell-derived exosomes as an alternative to adoptive cell transfer
}

\author{
Yeonsun Hong ${ }^{1}$ E In-San Kim ${ }^{1,2, *}$ \\ ${ }^{1}$ Biomedical Research Institute, Korea Institute of Science and Technology (KIST), Seoul 02792, ${ }^{2}$ KU-KIST Graduate School of Converging \\ Science and Technology, Korea University, Seoul 02841, Korea
}

\begin{abstract}
Adoptive cell transfer (ACT), a form of cell-based immunotherapy that eliminates cancer by restoring and strengthening the body's immune system, has revolutionized cancer treatment. ACT entails intravenous transfer of either tumor-resident or peripheral blood-modified immune cells into cancer patients to mediate anti-tumor response. Although these immune cells control and eradicate cancer via enhanced cytotoxicity against specific tumor antigens, several side effects have been frequently reported in clinical trials. Recently, exosomes, potential cellfree therapeutics, have emerged as an altemative to cell-based immunotherapies, due to their higher stability under same storage condition, lower risk of GvHD and CRS, and higher resistance to immunosuppressive tumor microenvironment. Exosomes, which are nano-sized lipid vesicles, are secreted by living cells, including immune cells. Exosomes contain proteins, lipids, and nucleic acids, and the functional role of each exosome is determined by the specific cargo derived from parental cells. Exosomes derived from cytotoxic effectors including $T$ cells and NK cells exert anti-tumor effects via proteins such as granzyme B and FasL. In this mini-review, we describe the current understanding of the ACT and immune cell-derived exosomes and discuss the limitations of ACT and the opportunities for immune cell-derived exosomes as immune therapies. [BMB Reports 2022; 55(1): 39-47]
\end{abstract}

\section{INTRODUCTION}

Cancer cells functionally design their microenvironment through the secretion of various factors such as cytokines and chemokines to maintain their proliferation and survival (1). In this process, immune cells are reprogrammed to undergo a drama-

${ }^{*}$ Corresponding author. Tel: +82-2-958-5935; Fax: +82-2-958-5609; E-mail: iskim14@kist.re.kr

https://doi.org/10.5483/BMBRep.2022.55.1.075

Received 24 May 2021, Revised 12 July 2021, Accepted 5 August 2021

Keywords: Adoptive cell transfer, Cancer, Exosome, Immune cell, Immunotherapy tic phenotypic change toward a pro-tumor profile, contributing to immune escape. Traditional cancer therapies, such as radiotherapy and chemotherapy, which target the tumor cells, initially induce positive responses in most patients, but is associated with frequent relapses and resistance $(2,3)$. Thus, immunotherapy, which utilizes the body's immune system to induce antitumor effects, is emerging as a useful tool (4-6).

The main goal of cancer immunotherapy is to boost and restore the anti-tumor immune response to eliminate cancer cells (7). Cancer immunotherapy includes adoptive cell transfer (ACT), checkpoint blockade, and anti-cancer vaccines (8). ACT utilizes $\mathrm{T}$ lymphocytes isolated from tumor tissues or genetically manipulated to recognize the specific antigen. The use of other immune cell types, such as natural killer (NK) cells, is also currently being studied. ACT of tumor-specific immune cells has proven clinical success in cancer treatment $(9,10)$. However, the challenges include autologous administration, auto-immune responses, off-tumor toxicity, and severe side effects such as cytokine release syndrome (CRS) (11).

Exosome-based cell-free therapy is emerging as a potential treatment to address these limitations of cell-based therapy. Exosomes are small endosomal derived, nano-sized lipid bilayer extracellular vesicles that carry a cargo of proteins, lipids, and nucleic acids $(12,13)$. Exosomes serve as important messengers that deliver functional cargo derived from parent cells to target cells and adjust the physiological or pathological processes of the target cells (14). NK cell-derived exosomes harboring FasL and NKG2D can mediate anti-tumor response (15). Also, dendritic cell (DC)-derived exosomes can induce an adaptive immune response by activating $\mathrm{CD} 4^{+} \mathrm{T}$ cells or $\mathrm{CD} 8^{+} \mathrm{T}$ cells via peptide-MHC complex (16).

Recently, several preclinical studies have been conducted to verify the anti-tumor effect of exosomes as immune therapeutics (17-19). Since exosomes are biocompatible with low cytotoxicity and immunogenicity, they can be utilized as carriers of biomarkers, vaccines, drugs, and therapeutics (19-22). In this review, we provide an inclusive overview of ACT and exosomes. Also, we discuss the challenges of ACT and the therapeutic potential of immune cell-derived exosomes in cancer immunotherapy.

ISSN: 1976-670X (electronic edition)

Copyright (C) 2022 by the The Korean Society for Biochemistry and Molecular Biology

(c) This is an open-access article distributed under the terms of the Creative Commons Attribution Non-Commercial License (http://creativecommons.org/licenses/by-nc/4.0) which permits unrestricted non-commercial use, distribution, and reproduction in any medium, provided the original work is properly cited. 


\section{ACT FOR CANCER IMMUNOTHERAPY}

ACT is a form of cell-based immunotherapy that uses immune cells to eliminate cancer $(10,23)$. ACT utilizes immune cells collected from patients selected or genetically engineered to express specific T-cell receptors (TCR) or chimeric antigen receptors (CAR). A sufficient number of immune cells expanded ex vivo, are infused into the patient. In this respect, $A C T$ has multiple advantages over other forms of cancer immunotherapy that rely on the in vivo development of sufficient numbers of anti-tumor immune cells (24). As shown in Fig. 1, ACT can be classified into four categories based on immune cell type and mechanism: tumor-infiltrating lymphocyte (TIL) therapy, engineered TCR therapy, CAR-T cell therapy and NK cell therapy.

\section{The types of ACT}

TIL therapy: TILs invading tumor tissues represent a heterogeneous population. TILs are primarily composed of $\mathrm{T}$ cells carrying a TCR capable of recognizing tumor-specific antigens and cytotoxic effects against tumors. TILs are emerging as important biomarkers for predicting the treatment outcome and efficacy. In the original TIL protocol, after isolating TILs from the tumor mass, a population of T cells with the desired TCR specificity can be selected and expanded in the presence of IL-2. These TILs are adaptively transferred to cancer patients via a lymphodepletion regimen.

Since TIL therapy was first administered to patients with metastatic melanoma in 1988 (25), it has been shown to be effective in many cancers, including melanoma, colon cancer, cholangiocarcinoma, and lung cancer (26). Currently, the clinical trials with autologous TIL (formally called LN-145) involve advanced cervical cancer (NCT03108495), metastatic non-small cell lung cancer (NSCLC) (NCT04614103), and triple-negative breast cancer (TBNC) (NCT04111510). Trials are also ongoing for combination therapy with TIL and chemotherapy (NCT
03467516) or cytokines (NCT01740557).

Engineered TCR therapy: Despite its clinical success, TIL therapy has limited availability and production of therapeutic $T$ cells for a larger group of patients. T cells genetically engineered to express TCR and CAR have been proposed as an effective alternative (27). TCR therapy entails the use of TCR-introduced $\mathrm{T}$ cells that can be linked to tumor antigens by extending the TIL therapy protocol. TCR is an $\alpha / \beta$ heterodimer composed of a constant region, which anchors into the $\mathrm{T}$ cell membrane, and a variable region, which recognizes and binds to the antigen-MHC complex.

In 2006, Morgan et al. reported the first successful clinical trials using autologous T cells with a TCR that was HLA-A2 restricted, and specific for the MART-1 antigen (28). A persistent clinical response was detected in 2 of 17 patients with refractory metastatic melanoma. In a follow-up study, treatment of patients with $\mathrm{T}$ cells expressing highly reactive TCRs against MART-1 resulted in tumor regression among 30\% of the cases (6 of 20) (29). Since then, Robbins PF et al. reported objective clinical responses of $60 \%$ and $45 \%$, in synovial cell sarcoma and melanoma patients, respectively, in a study using a TCR that recognizes the NY-ESO-1 antigen $(30,31)$. Currently, a clinical trial of NY-ESO-1-specific T cells in combination with chemotherapy including melphalan, for ovarian cancer, is underway (NCT03691376).

CAR-T cell therapy: Similar to TCR therapy, CAR-T therapy involves patient-derived T cells engineered to express chimeric antigen receptors (CARs) on the cell surface. CAR is a synthetic structure containing single-chain variable fragments (scFv) of a monoclonal antibody as the ligand-binding extracellular domain, a $\mathrm{CD} 3 \xi$ chain as the intracellular signaling domain and/or a co-stimulatory domain, mainly CD28 and 4-1BB $(32,33)$. Since CAR-T cells directly recognize surface antigens, but not the antigen presented by MHC, CAR-T cells can detect and attack cancer cells, unlike $T$ cells that fail to recognize cancer cells lacking MHC class in an evasion mechanism. CAR-T cells in

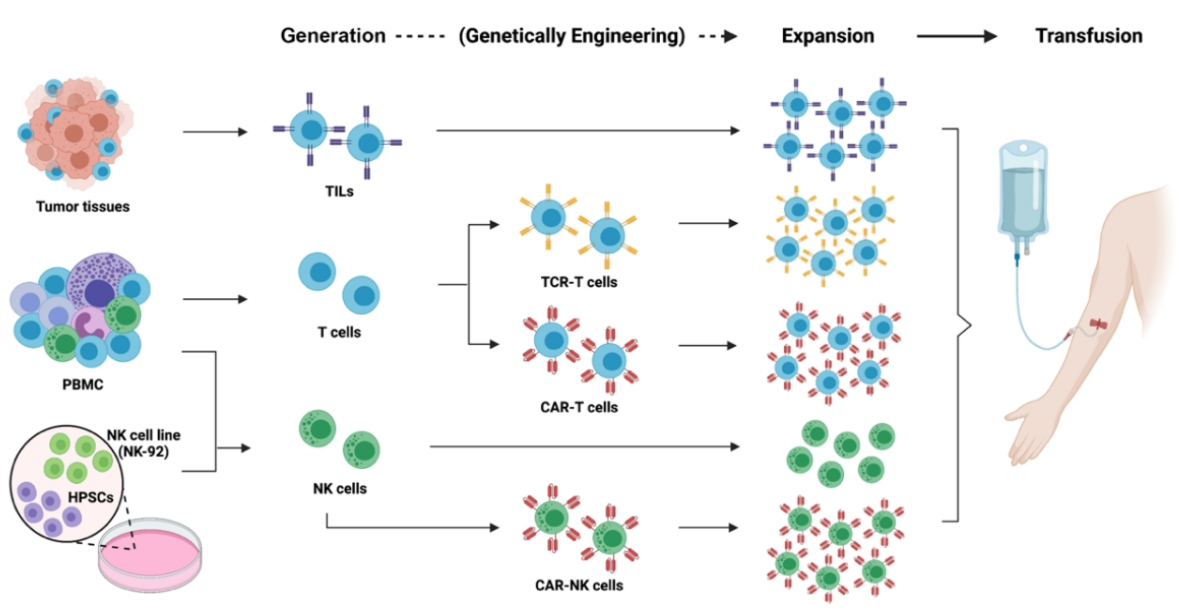

Fig. 1. Schematic diagram of adoptive cell therapy (ACT) process using $\mathrm{T}$ cells and NK cells. 
contact with cancer surface antigen proliferate and eliminate cancer cells via the release of effector molecules such as IFN- $\gamma$ and granzyme $\mathrm{B}$.

Most clinical studies have reported remarkable response rate against hematological neoplasms, such as acute lymphoblastic leukemia (ALL) and diffuse large B-cell lymphoma (DLBCL), suggesting the therapeutic potential of CD19-CAR-T therapy $(34,35)$. Since the first CAR-T therapies, Kymriah $^{(\mathbb{R}}$ (tisagenlecleucel) suspension for intravenous infusion for B-cell ALL was approved by the FDA in 2017, Yescarta ${ }^{\circledR}$ (axicabtageneciloleucel), Tecartus $^{\text {TM }}$ (brexucabtageneautoleucel), Abecma ${ }^{\text {TM }}$ (idecabtagenevicleucel), and Breyanzi ${ }^{\circledR}$ (lisocabtagenemaraleucel) were approved for lymphoma or myeloma (36).

NK cell therapy: NK cells, the innate immune cells, play an essential role in cancer immune surveillance (37). NK cells can quickly recognize and eliminate cancer cells without HLA matching or prior sensitization. NK cells are capable of killing target cells via a cytotoxic mechanism similar to that of $\mathrm{CD}^{+}$ cytotoxic T cells. Activated NK cells also mediate the innate and the adaptive immune system by releasing various inflammatory factors to recruit and activate other immune cells such as T cells and DCs. In addition, the NK cells are a significant factor predicting cancer prognosis (38).

Early approaches to NK cell therapy used fresh NK cells isolated from the patient's peripheral bold mononuclear cells (PBMCs) or whole blood (39). Because the number of NK cells in peripheral or cord blood is relatively low $(10-15 \%$ of all circulating lymphocytes), the use of NK cells for ACT required an ex vivo expansion mechanism to yield sufficient numbers of NK cells with high purity and potency. This challenge has recently been overcome via the differentiation of NK cells from pluripotent stem cells (PSCs), as well as the generation of NK-92 cell lines amenable to genetic manipulation for the recognition of specific tumor antigens (40). The use of antigenpresenting cells (APCs) as feeder cells in combination with CD137L-IL21 also enabled the production of a large number of activated NK cells. This success has enabled many clinical trials for NK cell-based cancer immunotherapy (41).

In addition, CAR-NK cells with improved anti-tumor activity than conventional NK cells have been developed using the basic structural framework of CAR designed for CAR-T cells (42). Many clinical trials are ongoing to evaluate the safety and efficacy of tumor-targeted CAR-NK cells. In the first clinical trial of CD33-CAR NK-92 cells in patients with relapsed and refractory acute myelogenous leukemia, there was no serious side effects showed when injected at doses of up to 5 billion cells per patient (NCT02944162) (43). In an ongoing CD19-targeted CAR-NK treatment clinical trial in patients with relapsed or refractory $\mathrm{CD} 19^{+}$cancer, about $73 \%$ of patients (8 of 11) manifested objective responses to treatment without major toxic effects (NCT03056339). Clinical trials of ROBO1 CAR-NK cells in solid tumors expressing ROBO1, including pancreatic cancer, are also ongoing (NCT03940820, and NCT03941457).

\section{Challenges of ACT therapy}

The remarkable success of ACT therapy is undeniable, but there are still many challenges to overcome. The ACT protocol involves the deletion of pre-lymphocytes and the infusion of live immune cells. Infused immune cells can cause graft-versushost disease (GvHD) by T cells that are not completely removed before treatment (44). Storage of expanded immune cells and reduction of their cytotoxicity and survival by freeze-thaw mechanisms should also be considered. The most common challenge is associated with toxicity including CRS induced by the immune cells used in ACT (45).

On-target off-tumor toxicity: Immune cells for ACT have been selected or genetically engineered to recognize tumor-specific antigens. However, the immune cells that target tumor antigens can also recognize healthy cells expressing the same antigen, causing "on-target off-tumor toxicity". For example, treatment of MART-1 specific or gp-100 specific T cells for melanoma exhibited toxicity in normal tissue including skin and ear in the presence of melanocytes (29). In patients with colorectal cancer treated with carcinoembryonic antigen (CEA)-specific TCR therapy, severe inflammatory colitis occurred due to CEA reactivity expressed in the normal colon epithelium (46). Similarly, the treatment for B cell malignancies by CD19 CAR-T cell induced B cell depletion and hypogammaglobulinemia (47). It has been reported that low levels of HER2 expression in normal lung tissue resulted in fatal lung toxicity in patients with metastatic colon cancer exposed to HER2 CAR-T therapy (48).

CRS: The most common toxicity induced by ACT involves CRS, a severe form called a cytokine storm (49). In immunotherapy, activated immune cells eliminate target tumors by releasing cytotoxic molecules including cytokines. CRS is mainly observed in CAR-T therapy due to the activation of the large number of $T$ cells injected and antigen recognition (50-52). CRS typically occurs within a few days following immune cell infusion and is associated with cell proliferation in vivo and a marked increase in the level of serum cytokines such as IFN- $\gamma$, TNF- $\alpha$, and IL-6 $(53,54)$. CRS is accompanied by symptoms such as fever, hypertension and hypoxemia, which can range from mild or moderate to life-threatening manifestations. A patient with metastatic colon cancer showed elevated levels of the serum cytokines including IFN- $\gamma$, TNF- $\alpha$, GM-CSF, IL- 6 and IL-10, after HER2 CAR-T treatment, eventually leading to death (48). In some patients (4 of 8) with B cell malignancies, excessive levels of serum cytokines (IFN- $\gamma$ and TNF- $\alpha$ ) were observed after CD19 CAR-T cell infusion (47).

Immune escape: Another challenge faced by ACT is that the tumor microenvironment (TME) depletes the anti-tumor function of the infused immune cells or interferes with their migration and penetration into solid tumors $(55,56)$. TME, composed of blood vessels, immune cells, extracellular matrix (ECM), and cancer cells, provides a milieu for tumor proliferation and progression. As infused T cells experience continued antigen stimulation and are exposed to immunosuppressive factors in 
TME, the T cells may be exhausted due to the loss of their effector function and up-regultion of inhibitory receptors such as PD-1 and Tim-3 (57). Moreover, the regulatory T cells or immunesuppressive modulators including prostaglandin E2 (PGE2), IL-6 and TGF- $\beta$ can suppress the cytotoxicity of infused NK cells (58-60). Solid tumors also prevent cytotoxic immune cells from migrating into or invading the tumor via secretion of chemokines or the formation of biological barriers such as ECM (61). Therefore, ACT for hematologic cancers has been effective, but the efficacy for solid cancers has room for improvement.

\section{IMMUNE CELL-DERIVED EXOSOMES FOR CANCER IMMUNOTHERAPY}

Despite its outstanding performance, ACT is limited by the direct use of immune cells. As mentioned above, several toxicities, including CRS and off-target effects, are triggered by uncontrolled immune cells in vivo. Moreover, current ACT strategies are limited in that they are costly and time-consuming to produce, preserve, and transport clinical-grade immune cells suitable for direct therapeutic use. Recent studies highlight the therapeutic potential and effectiveness of immune cell-derived exosomes as a cell-free immunotherapy.

\section{Overview of exosomes}

Exosomes are nano-sized membrane vesicles (30-150 nm) derived from various cell types including immune cells, tumor cells and mesenchymal stem cells (MSCs) $(62,63)$. Exosomes originate from endosomal pathway (62-64). Fusion of the multivesicular bodies (MVBs) generated by the inward budding of the late endosome with the cell membrane releases the intraluminal vesicles within into the extracellular space as exosomes. Exosomes are composed of proteins, nucleic acids, amino acids, metabolites and lipids (65). Exosomes are generally made up of many proteins involved in the biogenesis and function of exosomes including proteins associated with MVB biogenesis (Alix and TSG101), heat-shock protein (Hsp70) and the tetraspanins (CD9, CD63, CD81, and CD82) used as exosome markers. Exosomes also contain adhesion molecules such as ICAM-1 and integrins for cellular internalization (66). In addition, the exosomes contain an abundance of cholesterol, phosphatidylcholine, and diglycerides in the lipid rafts (67).

Although exosomes were initially considered as vesicles released to eliminate unnecessary contents, exosomes are involved in intracellular communication and represent a key factor regulating cellular function, especially the immune system $(68,69)$. Released exosomes can be present in diverse biological fluids such as milk, urine, and saliva, and delivered to target cells via blood and other body fluids (13). Also, exosomes are continuously released by donor cells, but their release is also controlled by cellular conditions, regulating the body's physiological responses (13).

\section{Immune cell-derived exosomes}

One of the earliest reported physiological targets of exosomemediated cell-to-cell communication is the immune system. In late 1990s, Raposo et al. demonstrated that exosomes secreted from B lymphocytes play a role in antigen presentation by inducing an antigen-specific $\mathrm{CD} 4^{+} \mathrm{T}$ cell response via a peptide-MHC class II complex on the surface (70). Subsequently, a variety of studies have reported the characteristics of exosomes derived from immune cells and their role in immune system. Immune cell-derived exosomes represent the functional properties of parental immune cells. APC-derived exosomes stimulate $\mathrm{CD}^{+}$and $\mathrm{CD}^{+}{ }^{+}$T cells via antigen-MHC complex expressed on their membrane (16).

Immune cell-derived exosomes express cell-specific marker proteins such as MHC class I and II and co-stimulatory molecules on APC-derived exosomes, CD56 on NK-derived exosomes and TCR/CD3 complex on T cell-derived exosomes (71). In addition, the tetraspanin family is abundant in immune cellderived exosomes (72). These proteins regulate the immune response by interacting with $\mathrm{MHC}$ molecules or cell adhesion molecules including LFA- 1 and ICAM-1 or by regulating the clustering of MHC complexes.

Clinical studies of exosomes derived from dendritic cells pulsed with tumor-specific peptides, as cancer vaccines, suggest the immunotherapeutic potential of immune cell-derived exosomes (73). Here, the anti-tumor functions of $\mathrm{T}$ cell-derived exosome and NK-derived exosome serving as an alternative to ACT are discussed in the following sections.

T cell-derived exosomes: $T$ cell-derived exosomes are produced only after T cells are activated (74). It was found that the interaction between tetraspanins, ceramides, and myelin and lymphocyte protein (MAL) proteins is important for the biogenesis of exosome by T cells. In particular, MAL protein, a tetraspanning membrane protein that is partly expressed in T cells, is involved in fusion of MVBs with the cell membrane (75). T cellderived exosomes strongly harbor TCR/CD3 complex and contain miRNAs and cytotoxic molecules including IFN- $\gamma$ and granzyme B. Exosomes originating from activated human $\mathrm{CD}^{+} \mathrm{T}$ cells, along with IL-2, induced the proliferation of resting $\mathrm{CD}^{+} \mathrm{T}$ cells and enhanced the level of cytokines and chemokines in the $\mathrm{CD}^{+}{ }^{+}$T cells (76). Li et al. confirmed that exosomes generated from cytotoxic T lymphocyte (CTL) stimulated with IL-12 contain enriched exosomal proteins such as Alix, CD9, CD81 and Tsg101 and CTL-associated proteins including granzyme B, STAT3, and STAT5B (77). Although these exosomes did not mediate memory CTL formation, they activated naïve $\mathrm{CD} 8{ }^{+} \mathrm{T}$ cells regardless of antigen, and reinforced the activation of CTLs under mild antigen stimulation. In addition, upon formation of immune synapse, miRNA-loaded exosomes were unidirectionally transferred from $\mathrm{T}$ cells to APCs (78). These results indicate that T cell-derived exosomes control the immune response.

Recently, Fu et al. reported that exosomes generated from CAR-T cell recognizing human EGFR and HER2 secreted 
cytotoxic effectors including granzyme B and perforin resulting in cytolytic activity and anti-tumor effects in xenograft models (79). In addition, CAR-T cell-derived exosomes injected into tumor-bearing mice did not express PD-1 on their membranes and did not induce CRS unlike CAR-T cells. Yang et al. demonstrated that exosomes derived from CAR-T cells targeting mesothelin, one of the antigens for breast cancer treatment, effectively inhibited cancer growth in TBNC animal models without apparent side effects via expression of perforin and granzyme B (80).

NK cell-derived exosomes: NK cells can kill abnormal cells such as cancer and stimulate adaptive immune response via secretion of pro-inflammatory cytokines and chemokines (37). Similar to parental cells, NK cell-derived exosomes express NK marker CD56 and receptors such as NKG2D that bind to ligands with restricted expression in malignant cells, and contain cytolytic molecules such as FasL, perforin and granzymes (15). In an early study of the NK cell-derived exosome, Lugini et al. reported that exosomes purified from NK cells expressed not only NK cell marker CD56, but also FasL and perforin, and showed cytolytic activity only in hematological cancer cells such as Jurkat and K562 cell lines (81). Later, it was demonstrated that exosomes isolated on a large scale from activated NK cells exerted cytotoxic activity against several cancer types including ALL and neuroblastoma via caspase-mediated pathway (82). These studies suggest that the activation of NK cells releases potent exosomes. Zhu et al. observed that NK cell-derived exosomes (NK-92 Exo) induced apoptosis in melanoma, but not normal cells and inhibited tumor growth in xenografts bearing melanoma cells (83). In addition, exosomes isolated from NK cells previously exposed to neuroblastoma (NB) cells carried NK cell receptors such as CD56 and NKG2D and exhibited anti-tumor effects against NB tumors in vitro and in vivo (84). NK cell-derived exosomes also induce cancer cell apoptosis via DNAX accessory molecule-1 (DNAM1) expressed on the surface (85). These results support the therapeutic potential of NK-derived exosome against cancer. Besides proteins including FasL and perforin, miRNA contained in NK-derived exosome is also involved in anti-tumor activity. Neviani et al. confirmed that exosomes derived from activated NK cells containing the tumor suppressor miR-186 displayed cytotoxicity against the MYCN-amplified NB cell line and restrained TGF- $\beta$ dependent immune escape (86).

Other immune cell-derived exosomes: DC-derived exosomes and macrophage-derived exosomes: In addition to exosomes derived from cytotoxic effectors that potentially kill tumor cells, exosomes derived from DCs or macrophages could serve as cancer vaccines. Exosomes isolated from tumor peptidepulsed DCs promote tumor-specific T cell priming by delivering MHC-restricted peptide loaded on the exosome surface to $\mathrm{T}$ cells or inducing expression of $\mathrm{MHC} /$ peptide complexes in DCs (16). Thus, like DC vaccines, DC-derived exosomes (DEXs) therapy can lead to tumor growth inhibition and tumor regression by inducing the patient's adaptive immune system to specific tumor antigens.

As a representative APC, DCs play an important role in mediating innate and adaptive immunity by recognizing, processing, and presenting antigens to T cells (87). Likewise, it has been demonstrated that DEXs can induce T cell priming by directly or indirectly presenting the MHC-antigen complex to T cells, and can also amplify $\mathrm{T}$ cell activation via co-stimulatory molecules such as CD86 and CD80 expressed on the surface (16). In addition, DEXs can facilliate activation and proliferation of NK cells via IL-15Ra ligand and NKG2D ligand (88). It was confirmed that TNF superfamily ligands (TNFSFLs) expressed on the DEX surface induce apoptosis in cancer cells and activate NK cells (89). Moreover, compared to DC-based vaccines, DEX immunotherapy has more resistance to tumor immunosuppression, higher bioavailability and biostability, with higher yields and lower costs (16). Based on these effects, DEXs have been used in several clinical trials investigating NSCLC (90, 91), metastatic melanoma (92) and colorectal cancer (93).

Among macrophage subtypes, M1-like macrophages can promote $\mathrm{T}$ cell-mediated immune responses by releasing cytokines such as IFN- $\gamma$, TNF- $\alpha$, IL-12 and IL- 6 or antigen presentation via phagocytosis. M1-like macrophage-derived exosomes, which retain immunostimulatory properties of parental cells can accelerate anti-cancer effects by releasing pro-inflammatory cytokines including IL-6, IL-12 and iNOS (94, 95). M1-like macrophage-derived exosomes containing anti-cancer drugs such as paclitaxel and cisplatin can enhance anti-cancer activity by inducing cancer cell apoptosis, increasing drug sensitivity and circumventing drug resistance mechanisms (95-98).

\section{Opportunities and challenges of immune cell-derived exosomes in immune therapies}

Recent studies have evaluated the role of exosomes derived from immune cells such as NK cells and T cells in immune modulation and their efficacy in preclinical studies. These results suggest that immune cell-derived exosomes display numerous functions suitable for clinical application. Furthermore, their advantages relate to storage and transplantation.

Stable storage of exosomes is an important issue in the transport and clinical application of exosomes. The storage techniques currently studied include cryopreservation, freeze-drying and spray drying. Cryopreservation $\left(-80^{\circ} \mathrm{C}\right.$ frozen storage) is a complete method for the stable storage of exosomes (99). The characteristics, function and efficacy of DEXs stored for a long time at $-80^{\circ} \mathrm{C}$ were not affected by freezing and thawing $(73,90,100)$. Further, the large number of exosomes isolated from NK cells expanded ex vivo were stable when stored at $-80^{\circ} \mathrm{C}$ for at least 12 months, and their cytotoxic effect was maintained (82). These results suggest that exosomes can be stably stored long term via cryopreservation.

Exosomes may be less toxic compared with cell-based therapies (ACT) that elicit serious immune responses such as $\mathrm{GvHD}$ or CRS. Morse et al. reported the treatment of patients with advanced NSCLC using DEXs without serious toxicity or 
autoimmune reactions, and no serious organ damage due to the vaccine occurred (90). In addition, Fu et al. reported that CAR-T cell-derived exosome did not induce changes in serum cytokine levels and body weight in mice, unlike CAR-T cells (79).

Despite the long-term storage of exosomes with their efficacy and properties intact, and low toxicity in ongoing clinical trials, currently no specific regulatory guidelines are available for clinical applications such as standardization, optimization and quality control. Given the heterogeneity and complexity of exosomes and most conventional laboratory-scale methods used for exosome isolation, it is difficult to isolate large volumes of exosomes with clinical grade-quality and purity (101). Recently, tangential flow filtration combined with a chromatographic method has been attempted to mass-produce high-quality exosomes that comply with good manufacturing practice (102-104). In addition, it has been proposed to use well-characterized cell lines instead of primary cells to ensure the uniformity and stability of exosomes (104).

\section{CONCLUSION AND PERSPECTIVES}

ACT entails the use of patient-derived immune cells such as T cells and NK cells that recognize tumor-specific antigens to eliminate cancers. Immune cells with unique cytotoxic effects show improved tumor recognition, continuous activation, and potent tumor killing capabilities through genetic engineering. ACT-based cancer immunotherapy has yielded promising results in clinical trials. Despite the encouraging results, many challenges remain. In vivo expansion and cytokine release of the infused immune cells induce adverse effects such as CRS and auto-immune responses. Also, the efficacy of ACT in solid cancers is limited due to poor cell migration and penetration into the tumor site, and immunosuppressive TME.

Exosomes derived from immune cells offer sufficient therapeutic potential as substitutes for ACT. Immune cell-derived exosomes exert immune-regulatory effects due to biofunctional cargo such as proteins and nucleic acids derived from their parental cells. Here, we highlight that exosomes isolated from cytotoxic effectors including T cells and NK cells exhibit antitumor effects identical to parental cells mediated via cytotoxic molecules such as FasL, IFN- $\gamma$ and perforin (Fig. 2). Further, the preclinical results provide evidence suggesting that these exosomes have low toxicity compared with $\operatorname{ACT}(79,80)$, which reinforces the exosome therapeutic potential.

Recent interest in exosomes worldwide has spurred research and production of exosomes for therapeutic purposes. The current state of exosome therapy is similar to early years of cell-based therapy limited by poor understanding of effective cell therapy, which hindered the large-scale development and manufature of specialized cells for treatment based on clinical studies. Further studies are needed to explore the possibility of immune cell-derived exosomes as immunotherapeutic agents. However, the results of existing preclinical studies demonstrate the poten- a)
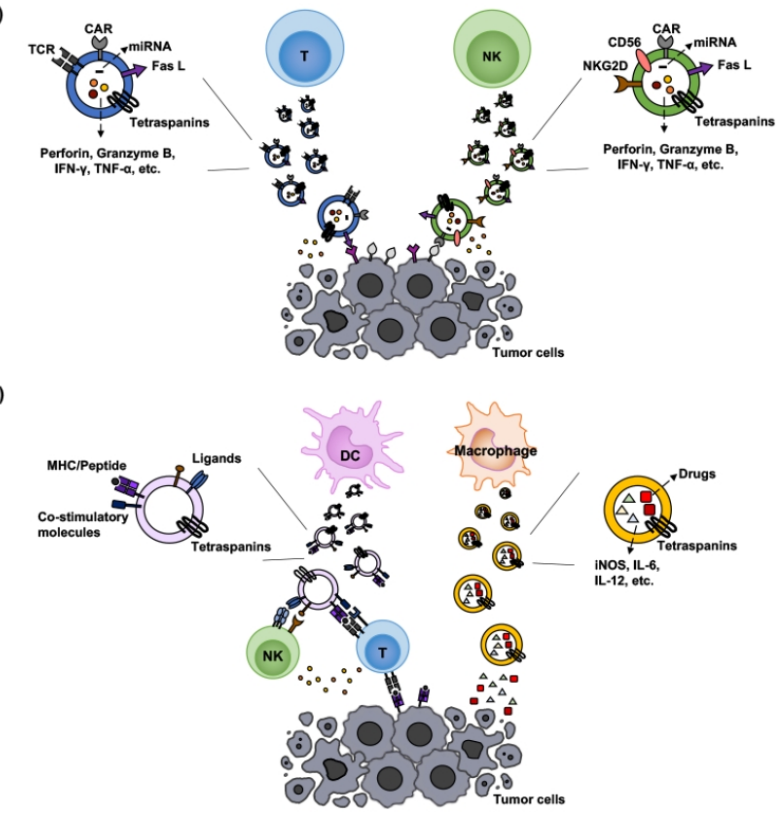

Fig. 2. Exosomes derived from immune cells. (A) T cell or NK cellderived exosomes that express FasL or NKG2D and contain cytotoxic molecules such as perforin, Granzyme B, IFN- $\gamma$, and TNF- $\alpha$ induce cancer cell death and inhibit cancer cell growth. (B) DCderived exosomes induce the activation of $\mathrm{T}$ cells and NK cells through the expression of some ligands such as NKG2D ligand, co-stimulatory molecules, and MHClantigen complex. Macrophagederived exosomes lead to cancer cell death by releasing pro-inflammatory cytokines such as iNOS, IL-6 and IL-12 or anti-cancer drugs.

tial of immune cell-derived exosomes as immunotherapeutics. Accordingly, exosomes derived from immune cells represent potential immunotherapeutic alternatives to ACT.

\section{ACKNOWLEDGEMENTS}

This work was supported by grants from the National Research Foundation of Korea (NRF) funded by the Korean government (2017R1A3B1023418), KU- KIST Graduate School of Convertging Science and Technology Program, and KIST Institutional Program.

\section{CONFLICTS OF INTEREST}

The authors have no conflicting interests.

\section{REFERENCES}

1. Hinshaw DC and Shevde LA (2019) the tumor microenvironment innately modulates cancer progression. Cancer Res 79, 4557-4566

2. Roma-Rodrigues C, Mendes R, Baptista PV and Fernandes 
AR (2019) Targeting tumor microenvironment for cancer therapy. Int J Mol Sci 20, 840

3. Baghban R, Roshangar $L$, Jahanban-Esfahlan $R$ et al (2020) Tumor microenvironment complexity and therapeutic implications at a glance. Cell Commun Signal 18, 59

4. Yu Y and Cui J (2018) Present and future of cancer immunotherapy: a tumor microenvironmental perspective. Oncol Lett 16, 4105-4113

5. Galluzzi L, Chan TA, Kroemer G, Wolchok JD and Lopez-Soto A (2018) The hallmarks of successful anticancer immunotherapy. Sci Transl Med 10, eaat7807

6. Chuah S and Chew V (2020) High-dimensional immuneprofiling in cancer: implications for immunotherapy. J Immunother Cancer 8, e000363

7. Disis ML (2014) Mechanism of action of immunotherapy. Semin Oncol 41 Suppl 5, S3-13

8. Houot R, Schultz LM, Marabelle A and Kohrt H (2015) T-cell-based immunotherapy: adoptive cell transfer and checkpoint inhibition. Cancer Immunol Res 3, 1115-1122

9. Rosenberg SA and Restifo NP (2015) Adoptive cell transfer as personalized immunotherapy for human cancer. Science 348, 62-68

10. Laskowski T and Rezvani K (2020) Adoptive cell therapy: Living drugs against cancer. J Exp Med 217, e20200377

11. Rohaan MW, Wilgenhof S and Haanen J (2019) Adoptive cellular therapies: the current landscape. Virchows Arch 474, 449-461

12. Kalluri R and LeBleu VS (2020) The biology, function, and biomedical applications of exosomes. Science 367, eaau6977

13. Zhang Y, Liu Y, Liu H and Tang WH (2019) Exosomes: biogenesis, biologic function and clinical potential. Cell Biosci 9, 19

14. Mathieu M, Martin-Jaular L, Lavieu G and Thery C (2019) Specificities of secretion and uptake of exosomes and other extracellular vesicles for cell-to-cell communication. Nat Cell Biol 21, 9-17

15. Fais S (2013) NK cell-released exosomes: natural nanobullets against tumors. Oncoimmunology 2, e22337

16. Pitt JM, Andre F, Amigorena S et al (2016) Dendritic cell-derived exosomes for cancer therapy. J Clin Invest $126,1224-1232$

17. Hong Y, Kim YK, Kim GB et al (2019) Degradation of tumour stromal hyaluronan by small extracellular vesicle$\mathrm{PH} 20$ stimulates CD103(+) dendritic cells and in combination with PD-L1 blockade boosts anti-tumour immunity. J Extracell Vesicles 8, 1670893

18. Kim GB, Nam GH, Hong Y et al (2020) Xenogenization of tumor cells by fusogenic exosomes in tumor microenvironment ignites and propagates antitumor immunity. Sci Adv 6, eaaz2083

19. Koh E, Lee EJ, Nam GH et al (2017) Exosome-SIRPalpha, a CD47 blockade increases cancer cell phagocytosis. Biomaterials 121, 121-129

20. Tran TH, Mattheolabakis G, Aldawsari $\mathrm{H}$ and Amiji $\mathrm{M}$ (2015) Exosomes as nanocarriers for immunotherapy of cancer and inflammatory diseases. Clin Immunol 160, 46-58

21. Yang Y, Hong Y, Cho E, Kim GB and Kim IS (2018)
Extracellular vesicles as a platform for membrane-associated therapeutic protein delivery. J Extracell Vesicles 7, 1440131

22. Hong $\mathrm{Y}, \mathrm{Nam} \mathrm{GH}$, Koh E et al (2018) Exosome as a vehicle for delivery of membrane protein therapeutics, $\mathrm{PH} 20$, for enhanced tumor penetration and antitumor efficacy. Adv Funct Mater 28, 1703074

23. Yang JC and Rosenberg SA (2016) Adoptive T-cell therapy for cancer. Adv Immunol 130, 279-294

24. Dudley ME and Rosenberg SA (2003) Adoptive-cell-transfer therapy for the treatment of patients with cancer. Nat Rev Cancer 3, 666-675

25. Rosenberg SA, Packard BS, Aebersold PM et al (1988) Use of tumor-infiltrating lymphocytes and interleukin-2 in the immunotherapy of patients with metastatic melanoma. A preliminary report. N Engl I Med 319, 1676-1680

26. Ott PA, Dotti G, Yee C and Goff SL (2019) An update on adoptive T-cell therapy and neoantigen vaccines. Am Soc Clin Oncol Educ Book 39, e70-e78

27. Essand M and Loskog AS (2013) Genetically engineered T cells for the treatment of cancer. J Intern Med 273, 166-181

28. Morgan RA, Dudley ME, Wunderlich JR et al (2006) Cancer regression in patients after transfer of genetically engineered lymphocytes. Science 314, 126-129

29. Johnson LA, Morgan RA, Dudley ME et al (2009) Gene therapy with human and mouse T-cell receptors mediates cancer regression and targets normal tissues expressing cognate antigen. Blood 114, 535-546

30. Robbins PF, Morgan RA, Feldman SA et al (2011) Tumor regression in patients with metastatic synovial cell sarcoma and melanoma using genetically engineered lymphocytes reactive with NY-ESO-1. J Clin Oncol 29, 917-924

31. Robbins PF, Kassim SH, Tran TL et al (2015) A pilot trial using lymphocytes genetically engineered with an NYESO-1-reactive T-cell receptor: long-term follow-up and correlates with response. Clin Cancer Res 21, 1019-1027

32. Miliotou AN and Papadopoulou LC (2018) CAR T-cell therapy: a new era in cancer immunotherapy. Curr Pharm Biotechnol 19, 5-18

33. Feins $\mathrm{S}$, Kong $\mathrm{W}$, Williams $\mathrm{EF}$, Milone $\mathrm{MC}$ and Fraietta JA (2019) An introduction to chimeric antigen receptor (CAR) T-cell immunotherapy for human cancer. Am J Hematol 94, S3-S9

34. Maude SL, Frey N, Shaw PA et al (2014) Chimeric antigen receptor $\mathrm{T}$ cells for sustained remissions in leukemia. N Engl J Med 371, 1507-1517

35. Schuster SJ, Svoboda J, Chong EA et al (2017) Chimeric antigen receptor $\mathrm{T}$ cells in refractory B-cell lymphomas. N Engl J Med 377, 2545-2554

36. Alexander M, Culos K, Roddy J et al (2021) Chimeric antigen receptor T-cell therapy: a comprehensive review of clinical efficacy, toxicity, and best practices for outpatient administration. Transplant Cell Ther 27, 558-570

37. Hu W, Wang G, Huang D, Sui M and Xu Y (2019) cancer immunotherapy based on natural killer cells: current progress and new opportunities. Front Immunol 10, 1205

38. Shimasaki N, Jain A and Campana D (2020) NK cells for cancer immunotherapy. Nat Rev Drug Discov 19, 200-218 
39. Liu S, Galat V, Galat Y, Lee YKA, Wainwright D and Wu J (2021) NK cell-based cancer immunotherapy: from basic biology to clinical development. J Hematol Oncol 14, 7

40. Klingemann $\mathrm{H}$, Boissel $L$ and Toneguzzo F (2016) Natural killer cells for immunotherapy - advantages of the NK-92 cell line over blood NK Cells. Front Immunol 7, 91

41. Kundu S, Gurney M and O'Dwyer M (2021) Generating natural killer cells for adoptive transfer: expanding horizons. Cytotherapy 23, 559-566

42. Bollino D and Webb TJ (2017) Chimeric antigen receptor-engineered natural killer and natural killer T cells for cancer immunotherapy. Transl Res 187, 32-43

43. Tang X, Yang L, Li Z et al (2018) First-in-man clinical trial of CAR NK-92 cells: safety test of CD33-CAR NK-92 cells in patients with relapsed and refractory acute myeloid leukemia. Am J Cancer Res 8, 1083-1089

44. Rapoport AP, Stadtmauer EA, Aqui N et al (2009) Rapid immune recovery and graft-versus-host disease-like engraftment syndrome following adoptive transfer of costimulated autologous T cells. Clin Cancer Res 15, 4499-4507

45. Magalhaes I, Carvalho-Queiroz C, Hartana CA et al (2019) Facing the future: challenges and opportunities in adoptive T cell therapy in cancer. Expert Opin Biol Ther $19,811-827$

46. Parkhurst MR, Yang JC, Langan RC et al (2011) T cells targeting carcinoembryonic antigen can mediate regression of metastatic colorectal cancer but induce severe transient colitis. Mol Ther 19, 620-626

47. Kochenderfer JN, Dudley ME, Feldman SA et al (2012) B-cell depletion and remissions of malignancy along with cytokine-associated toxicity in a clinical trial of anti-CD19 chimeric-antigen-receptor-transduced T cells. Blood 119, 2709-2720

48. Morgan RA, Yang JC, Kitano M, Dudley ME, Laurencot CM and Rosenberg SA (2010) Case report of a serious adverse event following the administration of $\mathrm{T}$ cells transduced with a chimeric antigen receptor recognizing ERBB2. Mol Ther 18, 843-851

49. Shimabukuro-Vornhagen $A$, Godel $P$, Subklewe $M$ et al (2018) Cytokine release syndrome. J Immunother Cancer 6, 56

50. Kochenderfer JN, Somerville RPT, Lu T et al (2017) Lymphoma remissions caused by anti-CD19 chimeric antigen receptor $\mathrm{T}$ cells are associated with high serum interleukin-15 levels. J Clin Oncol 35, 1803-1813

51. Neelapu SS, Tummala S, Kebriaei P et al (2018) Chimeric antigen receptor T-cell therapy - assessment and management of toxicities. Nat Rev Clin Oncol 15, 47-62

52. Bonifant $\mathrm{CL}$, Jackson $\mathrm{HJ}$, Brentjens RJ and Curran $\mathrm{KJ}$ (2016) Toxicity and management in CAR T-cell therapy. Mol Ther Oncolytics 3, 16011

53. Khadka RH, Sakemura R, Kenderian SS and Johnson AJ (2019) Management of cytokine release syndrome: an update on emerging antigen-specific $T$ cell engaging immunotherapies. Immunotherapy 11, 851-857

54. Murthy $\mathrm{H}$, lqbal $M$, Chavez JC and Kharfan-Dabaja MA (2019) Cytokine release syndrome: current perspectives. Immunotargets Ther 8, 43-52

55. Tang H, Qiao J and Fu YX (2016) Immunotherapy and tumor microenvironment. Cancer Lett 370, 85-90
56. Beavis PA, Slaney CY, Kershaw MH, Gyorki D, Neeson PJ and Darcy PK (2016) Reprogramming the tumor microenvironment to enhance adoptive cellular therapy. Semin Immunol 28, 64-72

57. Thommen DS and Schumacher TN (2018) T Cell dysfunction in cancer. Cancer Cell 33, 547-562

58. Slattery K and Gardiner CM (2019) NK cell metabolism and TGFbeta - implications for immunotherapy. Front Immunol 10, 2915

59. Riggan L, Shah S and O'Sullivan TE (2021) Arrested development: suppression of NK cell function in the tumor microenvironment. Clin Transl Immunology 10, e1238

60. Melaiu O, Lucarini V, Cifaldi L and Fruci D (2019) Influence of the tumor microenvironment on NK cell function in solid tumors. Front Immunol 10, 3038

61. Comoli P, Chabannon C, Koehl U et al (2019) Development of adaptive immune effector therapies in solid tumors. Ann Oncol 30, 1740-1750

62. Thery C, Zitvogel L and Amigorena S (2002) Exosomes: composition, biogenesis and function. Nat Rev Immunol 2, 569-579

63. Colombo M, Raposo G and Thery C (2014) Biogenesis, secretion, and intercellular interactions of exosomes and other extracellular vesicles. Annu Rev Cell Dev Biol 30, 255-289

64. Hessvik NP and Llorente A (2018) Current knowledge on exosome biogenesis and release. Cell Mol Life Sci $75,193-208$

65. Jeppesen DK, Fenix AM, Franklin JL et al (2019) Reassessment of exosome composition. Cell 177, 428-445 e418

66. Thery C, Ostrowski M and Segura E (2009) Membrane vesicles as conveyors of immune responses. Nat Rev Immunol 9, 581-593

67. Skotland T, Sandvig K and Llorente A (2017) Lipids in exosomes: current knowledge and the way forward. Prog Lipid Res 66, 30-41

68. Bobrie A, Colombo M, Raposo G and Thery C (2011) Exosome secretion: molecular mechanisms and roles in immune responses. Traffic 12, 1659-1668

69. Nam GH, Choi Y, Kim GB, Kim S, Kim SA and Kim IS (2020) Emerging prospects of exosomes for cancer treatment: from conventional therapy to immunotherapy. Adv Mater 32, e2002440

70. Raposo G, Nijman HW, Stoorvogel W et al (1996) B lymphocytes secrete antigen-presenting vesicles. J Exp Med 183, 1161-1172

71. Li Q, Wang $\mathrm{H}$, Peng $\mathrm{H}$, Huyan $\mathrm{T}$ and Cacalano NA (2019) Exosomes: versatile nano mediators of immune regulation. Cancers (Basel) 11, 1557

72. Jankovicova J, Secova P, Michalkova K and Antalikova J (2020) Tetraspanins, more than markers of extracellular vesicles in reproduction. Int J Mol Sci 21, 7568

73. Nikfarjam S, Rezaie J, Kashanchi F and Jafari R (2020) Dexosomes as a cell-free vaccine for cancer immunotherapy. J Exp Clin Cancer Res 39, 258

74. Ventimiglia LN and Alonso MA (2016) Biogenesis and function of T cell-derived exosomes. Front Cell Dev Biol 4,84 
75. Ventimiglia LN, Fernandez-Martin L, Martinez-Alonso E et al (2015) Cutting edge: regulation of exosome secretion by the integral MAL protein in T cells. J Immunol $195,810-814$

76. Wahlgren J, Karlson Tde L, Glader P, Telemo E and Valadi H (2012) Activated human T cells secrete exosomes that participate in IL-2 mediated immune response signaling. PLoS One 7, e49723

77. Li L, Jay SM, Wang Y, Wu SW and Xiao Z (2017) IL-12 stimulates CTLs to secrete exosomes capable of activating bystander CD8(+) T cells. Sci Rep 7, 13365

78. Mittelbrunn M, Gutierrez-Vazquez C, Villarroya-Beltri C et al (2011) Unidirectional transfer of microRNA-loaded exosomes from $\mathrm{T}$ cells to antigen-presenting cells. Nat Commun 2, 282

79. Fu W, Lei C, Liu S et al (2019) CAR exosomes derived from effector CAR-T cells have potent antitumour effects and low toxicity. Nat Commun 10, 4355

80. Yang $\mathrm{P}, \mathrm{Cao} \mathrm{X}, \mathrm{Cai} \mathrm{H}$ et al (2021) The exosomes derived from CAR-T cell efficiently target mesothelin and reduce triple-negative breast cancer growth. Cell Immunol 360, 104262

81. Lugini L, Cecchetti S, Huber V et al (2012) Immune surveillance properties of human NK cell-derived exosomes. J Immunol 189, 2833-2842

82. Jong $\mathrm{AY}, \mathrm{Wu} \mathrm{CH}$, Li J et al (2017) Large-scale isolation and cytotoxicity of extracellular vesicles derived from activated human natural killer cells. J Extracell Vesicles 6,1294368

83. Zhu L, Kalimuthu S, Gangadaran P et al (2017) Exosomes derived from natural killer cells exert therapeutic effect in melanoma. Theranostics 7, 2732-2745

84. Shoae-Hassani A, Hamidieh AA, Behfar M, Mohseni R, Mortazavi-Tabatabaei SA and Asgharzadeh S (2017) NK cell-derived exosomes from NK cells previously exposed to neuroblastoma cells augment the antitumor activity of cytokine-activated NK cells. J Immunother 40, 265-276

85. Di Pace AL, Tumino N, Besi F et al (2020) Characterization of human NK cell-derived exosomes: Role of DNAM1 receptor in exosome-mediated cytotoxicity against tumor. Cancers (Basel) 12, 661

86. Neviani P, Wise PM, Murtadha M et al (2019) Natural killer-derived exosomal miR-186 inhibits neuroblastoma growth and immune escape mechanisms. Cancer Res 79, 1151-1164

87. Wculek SK, Cueto FJ, Mujal AM, Melero I, Krummel MF and Sancho D (2020) Dendritic cells in cancer immunology and immunotherapy. Nat Rev Immunol 20, 7-24

88. Viaud S, Terme M, Flament $C$ et al (2009) Dendritic cell-derived exosomes promote natural killer cell activation and proliferation: a role for NKG2D ligands and IL-15Ralpha. PLoS One 4, e4942

89. Munich S, Sobo-Vujanovic A, Buchser WJ, Beer-Stolz D and Vujanovic NL (2012) Dendritic cell exosomes directly kill tumor cells and activate natural killer cells via TNF superfamily ligands. Oncoimmunology 1, 1074-1083

90. Morse MA, Garst J, Osada T et al (2005) A phase I study of dexosome immunotherapy in patients with advanced non-small cell lung cancer. J Transl Med 3, 9

91. Besse B, Charrier M, Lapierre V et al (2016) Dendritic cell-derived exosomes as maintenance immunotherapy after first line chemotherapy in NSCLC. Oncoimmunology 5, e1071008

92. Escudier B, Dorval T, Chaput $\mathrm{N}$ et al (2005) Vaccination of metastatic melanoma patients with autologous dendritic cell (DC) derived-exosomes: results of thefirst phase I clinical trial. J Transl Med 3, 10

93. Dai S, Wei D, Wu Z et al (2008) Phase I clinical trial of autologous ascites-derived exosomes combined with GM-CSF for colorectal cancer. Mol Ther 16, 782-790

94. Cheng L, Wang $Y$ and Huang L (2017) Exosomes from M1-polarized macrophages potentiate the cancer vaccine by creating a pro-inflammatory microenvironment in the lymph node. Mol Ther 25, 1665-1675

95. Wang P, Wang H, Huang Q et al (2019) Exosomes from M1-polarized macrophages enhance paclitaxel antitumor activity by activating macrophages-mediated inflammation. Theranostics 9, 1714-1727

96. Li J, Li N and Wang J (2020) M1 macrophage-derived exosome-encapsulated cisplatin can enhance its anti-lung cancer effect. Minerva Med [Online ahead of print]

97. Zhang X, Liu L, Tang M, Li H, Guo X and Yang X (2020) The effects of umbilical cord-derived macrophage exosomes loaded with cisplatin on the growth and drug resistance of ovarian cancer cells. Drug Dev Ind Pharm 46, 1150-1162

98. Kim MS, Haney MJ, Zhao Y et al (2016) Development of exosome-encapsulated paclitaxel to overcome MDR in cancer cells. Nanomedicine 12,655-664

99. Zhang Y, Bi J, Huang J, Tang Y, Du S and Li P (2020) Exosome: a review of its classification, isolation techniques, storage, diagnostic and targeted therapy applications. Int J Nanomedicine 15, 6917-6934

100. Tian Y, Li S, Song J et al (2014) A doxorubicin delivery platform using engineered natural membrane vesicle exosomes for targeted tumor therapy. Biomaterials 35, 2383-2390

101. Yang D, Zhang W, Zhang H et al (2020) Progress, opportunity, and perspective on exosome isolation - efforts for efficient exosome-based theranostics. Theranostics 10, 3684 3707

102. Busatto S, Vilanilam G, Ticer T et al (2018) Tangential flow filtration for highly efficient concentration of extracellular vesicles from large volumes of fluid. Cells 7, 273

103. Chen YS, Lin EY, Chiou TW and Harn HJ (2020) Exosomes in clinical trial and their production in compliance with good manufacturing practice. $\mathrm{Ci}$ Ji Yi Xue Za Zhi 32, 113-120

104. Whitford W and Guterstam P (2019) Exosome manufacturing status. Future Med Chem 11, 1225-1236 